\title{
Dependence of Effective Communication Distance and Characteristic Time on the Secretion Rate in Intercellular Signaling
}

\author{
Fukuo YosHIDA and Kihachiro HORIIKE* \\ Departments of Physics and * Biochemistry, Shiga University of Medical Science, Ohtsu, 520-2192 Japan
}

\begin{abstract}
Effective communication distance and characteristic time are discussed in relation to the temporal nonuniformity of the secretion rate in intercellular signaling. We demonstrated
\end{abstract}

that these characteristics significantly depend on secretion time and the strength of the enhanced secretion rate for human cytokines. [Japanese Journal of Physiology, 52, 399-401, 2002]

Key words: intercellular signaling, diffusion equation, secretion rate, temporal nonuniformity, communication distance.

F rancis and Palsson [1] have recently compared our models $[2,3]$ with their model [4] of effective communication distance and characteristic time in intercellular signaling. The effective communication distance and the characteristic time determine how far the signal can be transferred from a signaling cell to a target cell and the time required to complete this signal transfer, respectively. In this paper we highlight controversial points in Francis and Palsson's study and show that temporal nonuniformity plays an important role for these concepts in intercellular signaling by evaluating the concentration profile of secreted molecules.

Francis and Palsson [1] stated that we "modeled the cell as a constant spherical surface source" [2] in the same way they did [4]. In their model, the concentration $c(r, t)$ of the secreted molecules at any given time $t(>0)$ and at any given distance $r$ from the secreting cell that has a radius $a$ is given by Eq. 1 (i.e., eq. 2 of Francis and Palsson [4]);

$$
\begin{aligned}
c(r, t)= & \frac{F_{0} a}{r} \sqrt{\frac{t}{\pi D}\left[\exp \left\{-\frac{(r-a)^{2}}{4 D t}\right\}\right.} \\
& -\exp \left\{-\frac{(r+a)^{2}}{4 D t}\right\}-\sqrt{\frac{\pi}{4 D t}}|r-a| \operatorname{erfc}\left(\frac{|r-a|}{\sqrt{4 D t}}\right)
\end{aligned}
$$

$$
\left.+\sqrt{\frac{\pi}{4 D t}}(r+a) \operatorname{erfc}\left(\frac{r+a}{\sqrt{4 D t}}\right)\right]
$$

where $D$ is the diffusion constant of the signaling molecule and $F_{0}$ is its secretion rate. The complementary error function is represented by erfc. This function [5] is obtained by assuming a constant source of secreted molecules (e.g., proteins) on the cell surface. The "source" in their model refers to the production rate of the secreted molecules. On the other hand, we adopted the condition of a constant flux across the cell surface, or a constant secretion rate. The solution is given by Eq. 2 (i.e., eq. 10 of Yoshida and Horiike [2])

$$
\begin{aligned}
c(r, t)= & \frac{a^{2} j_{0}}{D r} \operatorname{erfc} \frac{r-a}{2 \sqrt{D t}} \\
& -\exp \frac{r}{a}-1+\frac{D t}{a^{2}} \operatorname{erfc} \frac{r-a}{2 \sqrt{D t}}+\frac{\sqrt{D t}}{a}
\end{aligned}
$$

where secretion rate is indicated by $j_{0}$. Consequently, it is not the source that is constant, but the flux across the cell surface, making Francis and Palsson's reference to the source in our model incorrect.

In Francis and Palsson's study, the source exists on

Received on May 7, 2002; accepted on July 30, 2002

Correspondence should be addressed to: Fukuo Yoshida, Department of Physics, Shiga University of Medical Science, Seta Tsukinowacho, Ohtsu, 520-2192 Japan. Tel and Fax: +81-77-548-2106, E-mail: fyoshida@belle.shiga-med.ac.jp 
the cell surface. Its necessary consequence that the secretion of signaling molecules from the cell surface in both inward and outward directions is physiologically incorrect. On the other hand, in our model the location of molecule production in the secreting cell and the intracellular transport to the cell surface are irrelevant. We only consider the number of molecules secreted per unit time from the cell surface to the surrounding medium, i.e., the flux across the cell surface or the secretion rate. A mechanism exists in the secreting cell to keep this secretion rate constant. In our model, which treats the cell as a black box, it is a fictitious source at its center [2], a situation analogous to the image-potential method used to solve boundary value problems in electrodynamics [6].

Francis and Palsson [1] stated that the time-dependency of the secretion rate can be ignored when the effective communication distance is being estimated. In contrast, we think that in models of intercellular signaling the secretion rate should be considered timedependent because paracrine secretion rates have been demonstrated by experiments to be time-dependent [7-9]. We considered this characteristic in our model [3] by introducing an enhanced secretion rate during the secretion time (secreting period) on the basis of a basal secretion level. This value is equivalent to the constant secretion rate if the secretion time is considered infinite. One of the important consequences of a finite secretion time is that the communication distance and characteristic time are not related to the molecule's saturation value, which is determined by using a stationary solution of the diffusion equation. In other words, the signal transfer is treated as a nonsaturated phenomenon by a signaling cell with an enhanced secretion rate and characteristic secretion time.

We examined the effects of a finite secretion time on the communication distance and of a characteristic time for human cytokines by assuming that the secretion rate exhibits "rectangular" time-dependency with an enhanced value $p_{1}$ during the time interval or the secretion time $t_{\mathrm{s}}$, decreasing to the basal level $p_{0}$ (see Yoshida et al. [3] for details). On the basis of our previous work [3], the values for the radius $a$ of the spherical secreting cell, the diffusion constant $D$ of the signaling molecule, and the dissociation constant of the signaling molecules and receptors in the target cells or the critical concentration $K$ were $10 \mu \mathrm{m}$, $5 \times 10^{-6} \mathrm{~cm}^{2} \mathrm{~s}^{-1}$, and $10 \mathrm{pM}$, respectively, for monoclonal antibody secretion from a myeloma cell [10]. The dimensionless (or reduced) concentration $\varphi(x, y)=a^{3} c\left(x a, a^{2} y / D\right)$ was determined by using Eq. 3 (i.e., eqs. 9 and 10 of Yoshida et al. [3]), in terms of the reduced length $x=r / a$ and the reduced time $y=D t / a^{2}$, as

$$
\varphi(x, y)=\frac{\beta_{1}}{x} f(x, y)+\theta\left(y-y_{\mathrm{s}}\right) \frac{\left(\beta_{0}-\beta_{1}\right)}{x} f\left(x, y-y_{\mathrm{s}}\right)
$$

with

$$
\begin{aligned}
f(x, y)= & \operatorname{erfc}\left(\frac{x-1}{2 \sqrt{y}}\right) \\
& -\exp (x-1+y) \operatorname{erfc}\left(\frac{x-1}{2 \sqrt{y}}+\sqrt{y}\right)
\end{aligned}
$$

where $\theta$ stands for the step function, $\beta_{1}=p_{1} a^{4} / D\left(\beta_{0}=\right.$ $\left.p_{0} a^{4} / D\right)$, and $y_{\mathrm{s}}=D t_{\mathrm{s}} / a^{2}$. The quantities $\beta_{1}\left(\beta_{0}\right)$ and $y_{\mathrm{s}}$ are the reduced values of the enhanced (basal) secretion rate $p_{1}\left(p_{0}\right)$ and the secretion time $t_{\mathrm{s}}$, respectively. We calculated $\varphi(x, y)$ by substituting $y_{\mathrm{s}}$, with $\beta_{1}=$ 4,800 and $\beta_{0}=0$ fixed. The results for the values of $y_{\mathrm{s}}=170\left(t_{\mathrm{s}}=34 \mathrm{~s}\right)$, its half value and twice larger value were shown in Table 1 . The value of $x_{\mathrm{c}}$ was derived to make the maximum value of $\varphi(x, y)$ as a function of $y$ equal to $2 \varphi_{\mathrm{c}}$, with $\varphi_{\mathrm{c}} \equiv K a^{3}=6$ being the reduced critical concentration. Also, $y_{1}$ and $y_{2}$ are the values of $y$ for which $\varphi\left(x_{\mathrm{c}}, y\right)=\varphi_{\mathrm{c}}$, and $y_{\mathrm{m}}$ represents the peak position of $\varphi(x, y)$ at the fixed distance $x$. The quantity $x_{\mathrm{c}}$ represents the value of the effective communication distance according to our fractional criteria, i.e., the procedure described above to determine $x_{\mathrm{c}}$ from $\varphi_{\mathrm{c}}$. During the time interval $y_{1} \leq y \leq y_{2}$, signal transfer is permitted as $\varphi\left(x_{\mathrm{c}}, y\right) \geq \varphi_{\mathrm{c}}$, and $y_{\mathrm{m}}$ is a representative value for the characteristic time. The concentration profiles corresponding to three cases of secretion time considered above at a representative distance, $x=30$ $(r=300 \mu \mathrm{m})$, from the secreting cell time are shown as a function of time in Fig. 1. The concentration profile and characteristic quantities were sensitive to the secretion time; by comparing the results at $y_{\mathrm{s}}=85$ and $y_{\mathrm{s}}=340$, the effective communication distance was shown to differ by more than $50 \%$, and the characteristic time increased three times. These results show

Table 1. Numerical values of the reduced distance $x_{\mathrm{c}}$, and the reduced times $y_{1}, y_{2}\left(>y_{1}\right), y_{m}$ obtained from three cases of $y_{s}$.

\begin{tabular}{rrrrr}
\hline$y_{\mathrm{s}}$ & $x_{\mathrm{c}}$ & $y_{1}$ & $y_{2}$ & $y_{\mathrm{m}}$ \\
\hline 85 & 31.4 & 115 & 566 & 210 \\
170 & 39.6 & 201 & 916 & 355 \\
340 & 49.7 & 352 & 1,495 & 604 \\
\hline
\end{tabular}

A clarification of the abbreviation is given in the text. 


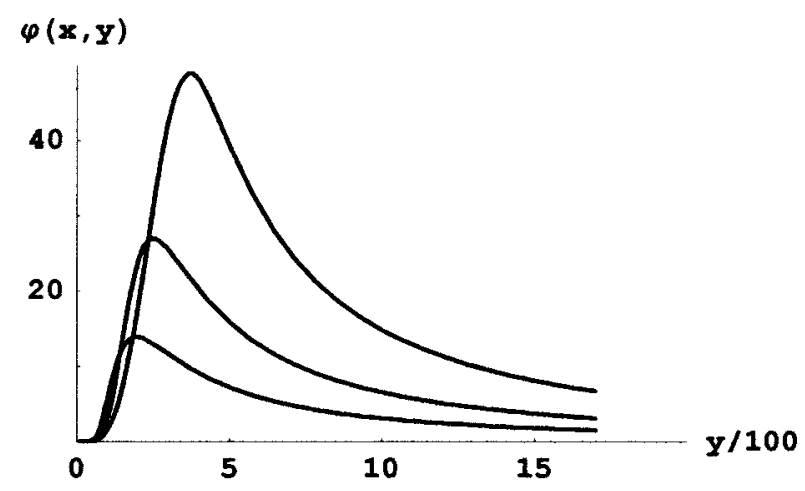

Fig. 1. Dependency of the concentration profile on secretion time. The three curves show a reduced concentration $\varphi(x, y)$ at the position with the reduced distance $x=30$, as a function of the reduced time $y / 100$, with the reduced secretion time $y_{s}=85,170$, and 340 , from bottom to top, respectively.

that the effects of temporal nonuniformity are significant for the realistic estimation of the effective communication distance and characteristic time.

In transforming growth factor $\alpha$ (TGF $\alpha)$ and epidermal growth factor (EGF), the input values were $a=4.92 \mu \mathrm{m}, D=9 \times 10^{-5} \mathrm{~cm}^{2} \mathrm{~s}^{-1}$, and $K=50 \mathrm{pM}$ [1]. We found that the unit of time was $a^{2} / D=2.7 \mathrm{~ms}$ and $\varphi_{\mathrm{c}}=3.6$, in comparison to $a^{2} / D=200 \mathrm{~ms}$ and $\varphi_{\mathrm{c}}=6$ in the first example. The value of $\varphi_{c}$ reduced approximately $50 \%$, but the secretion rate of $j_{0}=3.3 \times 10^{11}$ molecules $\mathrm{m}^{-2} \mathrm{~s}^{-1}$ is only about $10 \%$ of the corresponding value of $j_{0}=2.4 \times 10^{12}$ molecules $\mathrm{m}^{-2} \mathrm{~s}^{-1}$ in the first case. This indicates that much larger values than the value of 100 (or $p_{1}=100 j_{0}$ ) in the first example are required to realistically predict the effective communication distance [3]. It also implies that multiple cells contribute to cell signaling [1] because our models assume that cells independently secrete. We also investigated the effects of temporal nonuniformity by varying the secretion time. The results were qualitatively similar in reduced units to those shown above, with quantitative differences because of different input data.

In summary, we have clarified the differences between Francis and Palsson's [4] and our [2] models in response to the publication by Francis and Palsson [1]. The time-dependent diffusion equation is the same for both models, but there is a discrepancy over whether cell signaling exhibits a production rate or a secretion rate. We highlighted that the models have different underlying physicochemical pictures and that their model is physiologically incorrect.
We also demonstrated that models should not assume the presence of a constant secretion rate. Incorporating time-dependency into the model does not introduce unnecessary complexity, but it is an essential component of the model [3] as signal transfers take place within a finite time, and models should be able to explain this phenomenon within its framework. Using a quantitative analysis of concentration profiles, we demonstrated a significant contribution from time dependency. For example, the effective communication distance of monoclonal antibody secretion from a myeloma cell increased from about $300 \mu \mathrm{m}\left(x_{\mathrm{c}}=\right.$ 31.4 ), or 15 cell diameters, for the secreting period $t_{\mathrm{s}}=17 \mathrm{~s}\left(y_{\mathrm{s}}=85\right)$ to about $500 \mu \mathrm{m}\left(x_{\mathrm{c}}=49.7\right)$, or 25 cell diameters, for $t_{\mathrm{s}}=68 \mathrm{~s}\left(y_{\mathrm{s}}=340\right)$. We conclude that this temporal nonuniformity of secretion rate is important to clarify the effective communication distance and characteristic time in intercellular signaling.

\section{REFERENCES}

1. Francis $\mathrm{K}$ and Palsson B: Comment on "Time dependent concentration profile of secreted molecules in the intercellular signaling." J Phys Soc Jpn 71: 1005-1006, 2002

2. Yoshida $\mathrm{F}$ and Horiike $\mathrm{K}$ : Communication distance in the intercellular signaling by cyto/chemokine or autacoid. Proc Jpn Acad 75B: 87-91, 1999

3. Yoshida F, Horiike $\mathrm{K}$, and ShiPing $\mathrm{H}$ : Time-dependent concentration profile of secreted molecules in the intercellular signaling. J Phys Soc Jpn 69: 3736-3743, 2000

4. Francis $\mathrm{K}$ and Palsson BO: Effective intercellular communication distances are determined by the relative time constants for cyto/chemokine secretion and diffusion. Proc Natl Acad Sci USA 94: 12258-12261, 1997

5. Carslaw HS and Jaeger JC: Conduction of Heat in Solids, 2nd ed, Oxford University Press, New York, pp 261-263, 1959

6. Jackson JDE: Classical Electrodynamics, John Wiley \& Sons, New York, 2nd ed, pp 54-78, 1975

7. Alberts B, Bray D, Lewis J, Raff M, Roberts K, and Watson JD: Molecular Biology of the Cell, 3rd ed, Garland Publishing, New York, pp 599-651, 721-785, 1994

8. Ciolkowski EL, Cooper BR, Jankowski JA, Jorgenson JW, and Rightman RM: Direct observation of epinephrine and norepinephrine cosecretion from individual adrenal medullary chromaffin cells. J Am Chem Soc 114: 2815-2821, 1992

9. Brock TG, McNish RW, and Peters-Golden M: Arachidonic acid is preferentially metabolized by cyclooxygenase-2 to prostacyclin and prostaglandin $\mathrm{E}_{2}$. J Biol Chem 274: 11660-11666, 1999

10. Savinell JM, Lee GM, and Palsson BO: On the orders of magnitude of epigenic dynamics and monoclonal antibody production. Bioprocess Eng 4: 231-234, 1989 